\title{
Air Shower Measurement with a SiPM based Scintillator Detector
}

\author{
J. Kemp*, T. Bretz, T. Hebbeker, L. Middendorf, C. Peters and J. Schumacher \\ Physics Institute III A \\ RWTH Aachen University, Aachen \\ E-mail: kemp@physik.rwth-aachen.de
}

We developed an optical module based on silicon photomultipliers (SiPMs) designed for a wide variety of applications especially in scintillator detectors and laboratory measurements. To test the module, a Scintillator Surface Detector developed for AugerPrime, the upgrade of the Pierre Auger Observatory, was modified and is operating in the Argentinean Pampas. It is the first SiPM based scintillator detector successfully measuring air showers induced by ultra-high energy cosmic rays.

The main goal of the development was exploiting the advantages of SiPMs compared to conventional photomultiplier tubes, specifically their mechanical and optical robustness, their high photon detection efficiency and their lower operating voltage.

Two SiPMs are read out in parallel and equipped with light guides to increase the sensitive area. Their signal is split into three independent gain stages to exploit the full dynamic range from a breakdown of a single SiPM cell to a simultaneous breakdown of all cells. The bias voltage supply, which corrects for the temperature, is integrated into the module, and it is powered and controlled through a USB connection. This setup guarantees an easy usage of the module in many applications.

We present the module and will show results from the field measurements.

36th International Cosmic Ray Conference -ICRC2019-

July 24th - August 1st, 2019

Madison, WI, U.S.A.

\footnotetext{
* Speaker.
} 


\section{Introduction}

To detect cosmic ray induced air showers with ground based detectors, there are several different measurement techniques currently in operation. The fluorescence or Cherenkov light produced by charged particles in the atmosphere can be observed by telescopes looking into the sky (e.g. the Pierre Auger Observatory [1], MAGIC [2], FAMOUS [3]). These telescopes can only be operated during clear moonless nights reducing the duty cycle. A duty cycle of close to $100 \%$ can be achieved using particle detectors measuring the shower footprint at ground. A common method used in many experiments is to distribute water or ice filled tanks (e.g. the Pierre Auger Observatory [4], HAWC [5], IceTop [6]) or use scintillator detectors (e.g. Telescope Array [7]). Radio arrays (e.g. AERA [8]) allow for a high duty cycle and a measurement of the electromagnetic component of air showers.

Except for the radio arrays, all of these detectors have in common that very faint light fluxes have to be detected. For many decades, the main light detection device used in these applications was the photomultiplier tube (PMT) as it allows for single photon detection. In recent decades, the semiconductor industry has made huge progress also developing sensitive and precise light sensors. Typical examples are CCD- or CMOS-sensors used in digital photo cameras. To measure low light fluxes avalanche photo-diodes (APDs) can be used. By operating them in Geiger-mode (G-APD) above their breakdown voltage, they even allow for single photon detection with high sensitivity. In order to allow for large sensitive areas and a high dynamic range, multiple G-APDs can be operated in parallel resulting in a silicon photomultiplier (SiPM). They are typically compiled of one hundred up to several ten thousand G-APDs. Compared to conventional PMTs, they are optically and mechanically very robust and have a similar or higher photon detection efficiency.

These characteristics make them ideal candidates for the development of new cosmic ray detectors.

\section{Silicon Photomultipliers}

Silicon photomultipliers (SiPMs) are semiconductor based light sensors compiled of G-APDs. While conventional PMTs have to be operated at $\sim 1000 \mathrm{~V}$, SiPMs are operated with voltages below $100 \mathrm{~V}$ allowing for a simpler power supply. The operating voltage for recent Hamamatsu devices is in the range of 50 to $60 \mathrm{~V}$ and even below $30 \mathrm{~V}$ for SensL ${ }^{1}$ devices. Most characteristics of SiPMs depend on the overvoltage $V_{o v}$ which is the excess of the bias voltage $V_{b}$ above the breakdown voltage $V_{b r}$. It is usually in the range of a few volts. The gain of the SiPM shows a linear dependence on the overvoltage and is in the order of $10^{6}$ [9], while the photon detection efficiency (PDE) shows a slower increase. In addition, also the dark count rate increases with the overvoltage. Thus, a compromise has to be found between a low dark count rate and a high PDE and gain.

The breakdown voltage is temperature dependent. To allow for a stable operation also under changing ambient temperatures, the bias voltage has to be adjusted. This is of major importance for air shower experiments as they are typically operated outside throughout the whole year. For recent Hamamatsu devices, the change of the breakdown voltage is $\sim 60 \mathrm{mV} / \mathrm{K}$. Thus, for typical

\footnotetext{
${ }^{1}$ Now part of ON Semiconductors
} 
temperature variations of $60 \mathrm{~K}$ throughout the year, this can lead to significant variations in the SiPM characteristics. Typically, bias voltage supplies optimized for the operation of SiPMs allow for monitoring the ambient temperature and adjust the bias voltage automatically to allow stable operation.

The noise of SiPMs originates from different sources. The major component originates from thermal excitations in the silicon lattice initiating a breakdown of a cell. It occurs randomly in time and is strongly temperature dependent. As random coincidences are rare, it is mainly on the level of one photon equivalent (p.e.). A second component is optical crosstalk. It is induced by optical photons produced during the breakdown of a cell. These photons can trigger neighboring cells. For recent devices this effect occurs in a few percent of the breakdowns [10]. A final noise effect is afterpulsing. It originates from electrons or holes that are trapped by impurities of the lattice structure during an avalanche. They are released with a delay inducing an additional avalanche. As recent devices only have very few impurities, the afterpulsing probability is negligible.

\section{A General Purpose SiPM based Optical Module}

To exploit the advantages of SiPMs we developed an SiPM based optical module optimized for the application in scintillator detectors but also suitable for a wide range of applications [11]. A photo and a schematic overview are shown in figure 1 . The module consists of two printed circuit

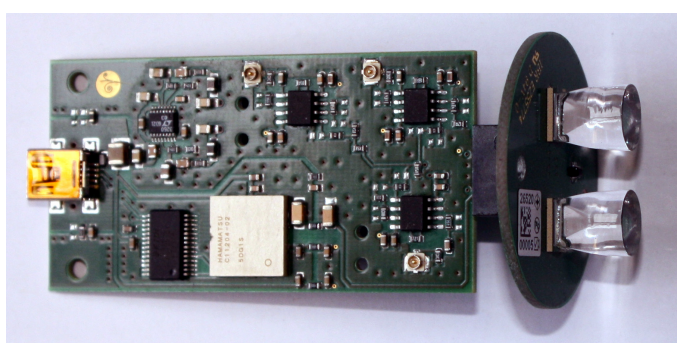

(a)

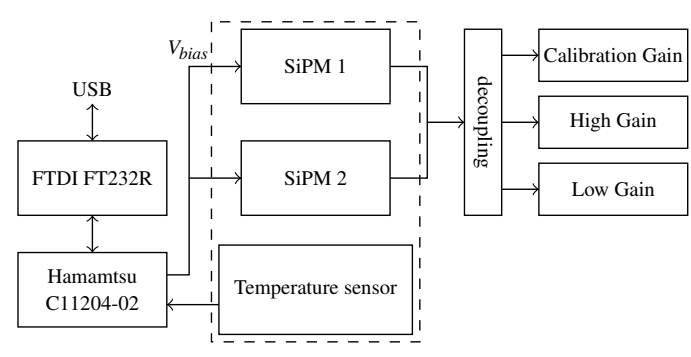

(b)

Figure 1: left: A picture of the module. It is split in two PCBs. One PCB hosts two SiPMs [10] equipped with light guides and a temperature sensor. The second PCB hosts three independent gain stages, the power supply [12] and digital communication. It has roughly credit card size. right: A schematic overview of the module.

boards (PCBs). The first PCB holds two SiPMs of type Hamamatsu S13360-6025PE [10] with a surface area of $6 \times 6 \mathrm{~mm}^{2}$ each and a cell pitch of $25 \mu \mathrm{m}$. The rather small cell size allows for a high number of cells and thus a high dynamic range. To increase the effective area, acrylic glass light guides optimized for the readout of wavelength shifting fibers are glued on the SiPMs. In order to be able to exploit the full dynamic range of the SiPMs from the breakdown of a single cell (one photoelectron equivalent (p.e.)) to a simultaneous breakdown of all cells corresponding to 115,200 p.e. the signal is split into three different amplifiers with different gain. In the following, the three different channels will be referred to as (from highest to lowest gain) calibration gain $(C G)$, high gain $(H G)$, and low gain $(L G)$.

The power supply is realized through a Hamamatsu C11204-02 [12] integrated circuit. It allows for automatic adjustment of the bias voltage according to the ambient temperature. The 
temperature is not measured on the chip but on the PCB holding the SiPMs. Communication and power is supplied through a mini USB type B connector allowing for operation with any standard USB device. Thus, the module is also well suited for laboratory applications. Through the USB connection, the bias voltage as well as the temperature correction coefficient can be set. In addition, set voltage, monitored voltage, monitored current, and monitored temperature can be read out.

Details on the developed electronics as well as the optical components can be found in [11].

\subsection{The Pierre Auger Observatory}

The Pierre Auger Observatory [13] located in the Argentinean Pampas measures cosmic ray induced air showers on an instrumented area of $3,000 \mathrm{~km}^{2}$ with a hybrid detector. A fluorescence detector (FD [1]) that consists of 27 telescopes measures the longitudinal development of air showers in the atmosphere during clear moonless nights. In addition, a surface detector (SD [4]) of 1,660 waver-Cherenkov detector stations (WCDs) is placed on a hexagonal grid. 1,600 of those stations have a spacing of $1,500 \mathrm{~m}$ while the other 60 stations reduce the spacing in a smaller area down to $750 \mathrm{~m}$ and $433 \mathrm{~m}$, which lowers the energy threshold. Each WCD contains $12 \mathrm{~m}^{3}$ of purified water and is equipped with three PMTs detecting the Cherenkov light produced by through going charged particles.

\subsection{The Scintillator Surface Detector}

The Scintillator Surface Detector (SSD) [14] has been developed by the Pierre Auger Collaboration in order to upgrade their observatory within the scope of the AugerPrime project [15]. On top of each WCD one SSD will be placed. A SSD consists of 48 scintillator bars with a size of $160 \times 5 \times 1 \mathrm{~cm}^{3}$ each. They are arranged in two halves and the light is collected by 48 wavelength shifting (WLS) fibers guiding it to the optical module. In the baseline design it is one PMT placed between the two detector halves. As the detector is well suited also for a usage with SiPMs, in total four of them have a slightly modified design to allow operation and testing also with the presented SiPM module. For these detectors, the ends of the WLS fibers are arranged in two bundles instead of one to match the geometry of the SiPM module. Two of these detectors are installed at the Pierre Auger Observatory in the Argentinean Pampas and allow for the measurement of cosmic ray induced air showers. One of the detectors (ID 59) is installed in the regular grid of the Pierre Auger Observatory with 1,500 m spacing. The other detector (ID 41) is located in a region of denser grid spacing of only $433 \mathrm{~m}$ allowing for a higher event rate. For both detectors, at least one regular surface detector station and one upgraded surface detector station equipped with a SSD of baseline design are located only a few meters apart. This setup allows for precise comparison of the different detectors when measuring the same air shower.

\section{Calibration of the SiPM based Optical Module}

The response of the SSDs is calibrated by measuring the signal produced by a through-going minimum ionizing particle (MIP). The PMTs of the WCD trigger the readout of a signal trace when a muon is detected. The corresponding trace is integrated and the total signal filled into a charge histogram. For the SiPM based optical module, the histogram is taken using the signals from the CG or HG in an alternating way. Examples of the two histograms are shown in figure 2. 


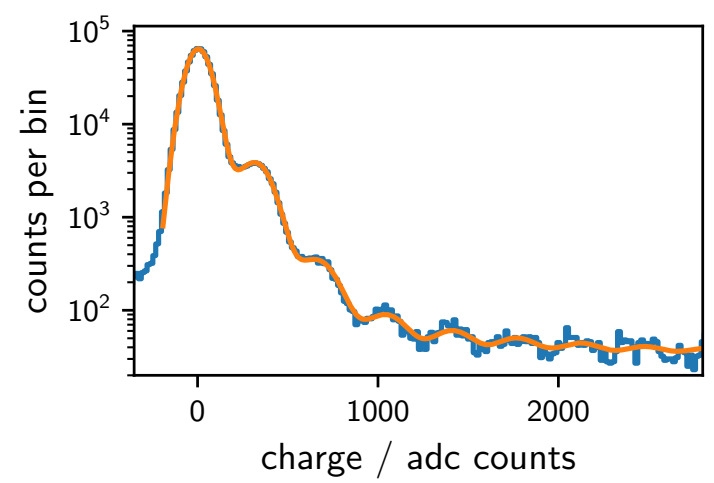

(a)

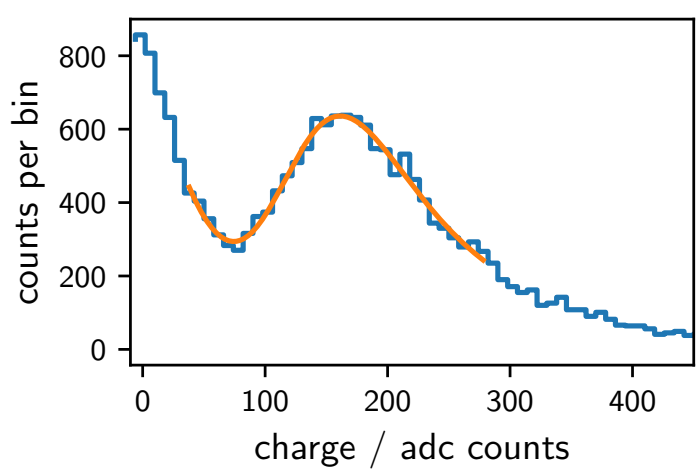

(b)

Figure 2: Calibration histograms determined using signals from the CG (left) and HG (right) channels, respectively. The histogram of the CG channel is zoomed into the pedestal region. It allows for resolution of single p.e.. The signal distribution in both cases is fitted using an exponential to describe background and a Landau distribution folded with a Gaussian to describe the MIP peak. For the CG, a sum of correlated modified Gaussians is used to describe the single p.e. peaks.

The calibration of the module is possible in both cases and the MIP peak is well separated from the pedestal. Thus, precise calibration of the signals is possible. For the CG channel, the single p.e. peaks are visible and their mutual distance can be determined. It is proportional to the gain of the SiPM and thus allows for continuous monitoring.

\subsection{Temperature stability of the calibration}

As described in section 2, the breakdown voltage of SiPMs is temperature dependent. The presented optical module adjusts the bias voltage according to temperature to keep the breakdown voltage stable. In figure 3 the gain and the position of the MIP peak for the CG channel are shown with respect to temperature. The temperature measured at the SiPM module is not transmitted in the normal data stream. Instead, the temperature of one of the WCD PMTs is used here. The gain of the SiPMs is very stable for temperatures above $20^{\circ} \mathrm{C}$. At lower temperatures a slight decrease in the gain can be seen. It might only be a systematic of the fit but can only be properly studied when data also at lower temperatures is available. The position of the MIP peak is temperature dependent. As the gain of the SiPM is stable and thus is its overvoltage, it can be concluded that this dependency arises from the optical components of the detector such as the scintillator or WLS fiber.

\subsection{Cross calibration of the signal channels}

The optical module hosts three signal channels. Their gain ratio is calibrated using signals that do not saturate the analog to digital converter (ADC) of the readout electronics. In figure 4, two calibration curves for CG and HG channel and HG and LG channel, respectively, are shown. The signal is determined by integrating the measured traces. The channels have good overlap that allows for a precise cross calibration. 


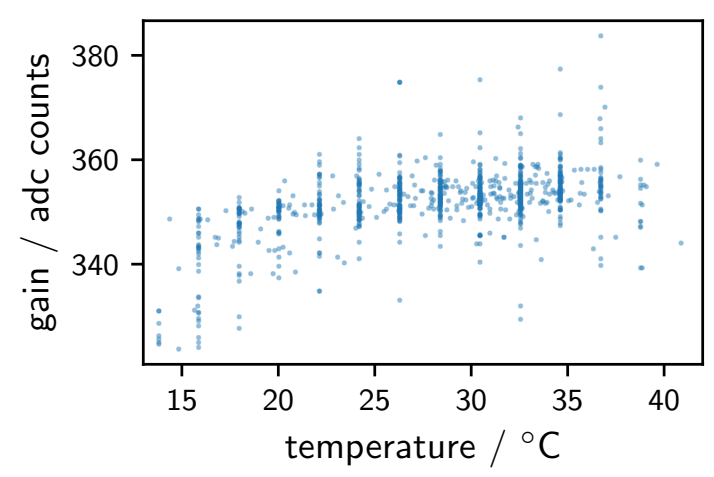

(a)

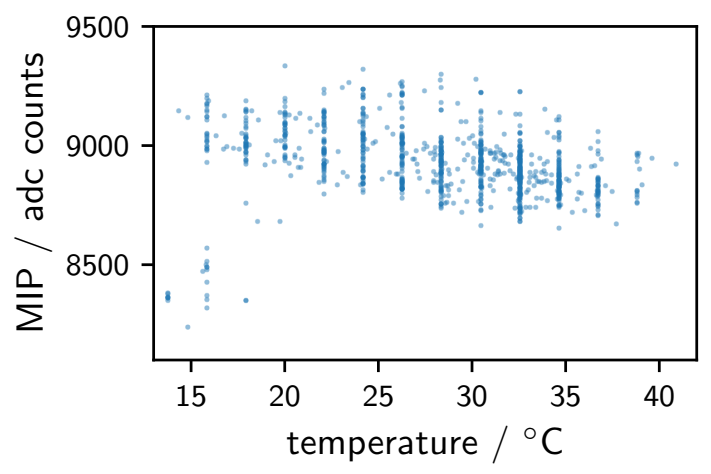

(b)

Figure 3: Gain (left) and position of the MIP peak (right) with respect to temperature. As the temperature data from the SiPM module is not stored, the temperature from one of the WCD PMTs is used here. Vertical lines originate from the resolution of the temperature measurement. Intermediate points originate from interpolating between neighboring measurements if no temperature measurement was available. The gain is very stable for temperatures above $20^{\circ} \mathrm{C}$ while the MIP peak shows a decrease with increasing temperature. It originates from a varying efficiency of the scintillator or WLS fiber.

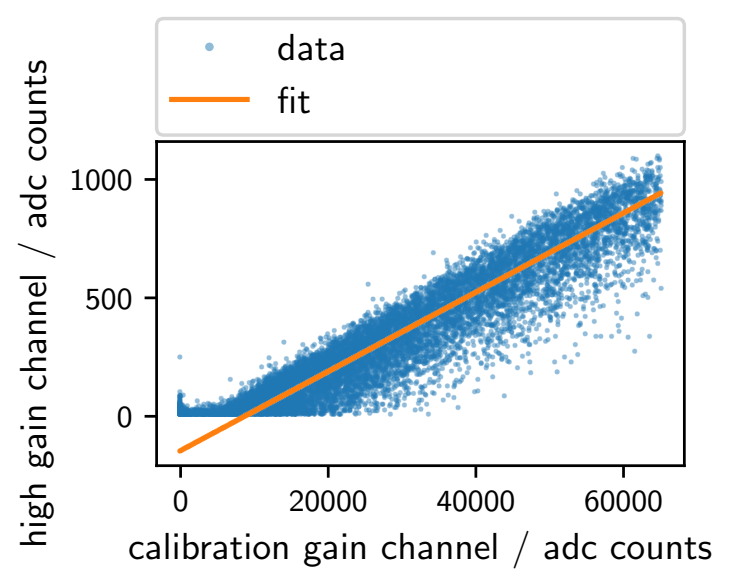

(a)

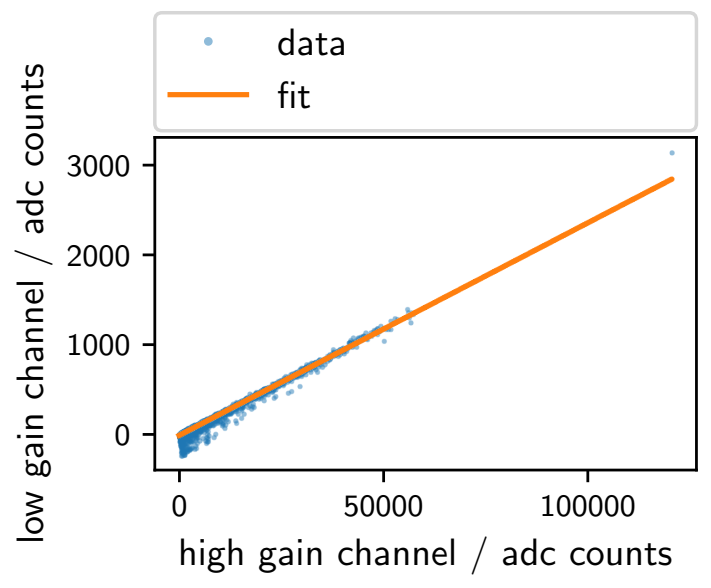

(b)

Figure 4: Cross calibration of the CG and HG channels (left) and the HG and LG channels (right). In both cases, a good overlap between the two channels assures precise calibration. The fit is performed to the median of the distributions. In the left case, events above 60,000 adc counts in the CG channel are cut away as they suffer from saturation effects. 


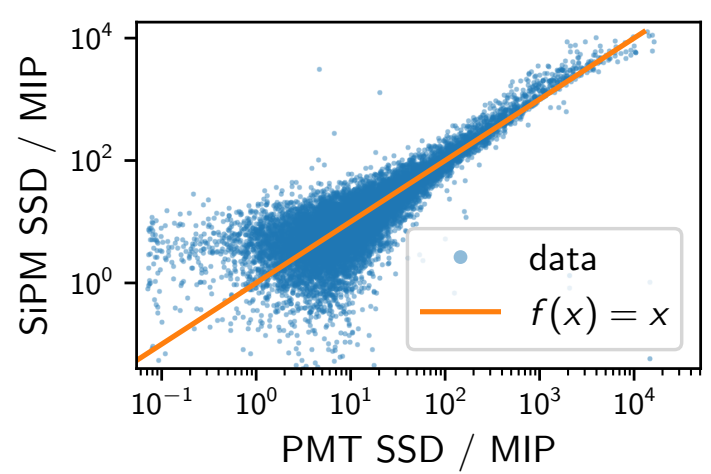

(a)

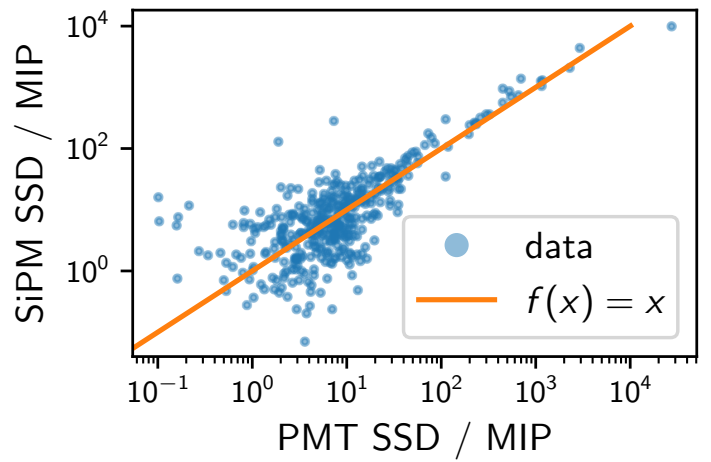

(b)

Figure 5: Comparison of the calibrated signal measured in two neighboring detectors. left: Detector ID 41 (SiPM) compared to detector ID 22 (PMT). right: Detector ID 59 (SiPM) compared to detector ID 56 (PMT). The detectors shown in the left plot are placed in a region with denser grid spacing of the array. Thus, also lower energy showers are triggered compared to the standard array. The higher statistics allows for a precise comparison of the detectors. The detectors shown in the right plot are placed in the standard array. Thus, the trigger rate is lower.

\section{Comparison between neighboring SSDs}

As already has been mentioned in section 3.2, for each of the SSDs equipped with the SiPM based optical module, another SSD equipped with a PMT is located only a few meters apart. This setup allows for a precise comparison of the signal measured in the two detectors. The results of comparing the signal in neighboring detectors are shown in figure 5. For low signals, the spread of the distributions is rather wide. It is a result of the different locations of the two detectors and expected when measuring the same air shower at slightly different positions. The correlation between the signals of the SSDs equipped with the SiPM based optical module and the PMT is very good even up to signals of about 10,000 MIP. For higher signals, the SiPMs begin to show effects of non-linear behavior. These could be corrected for using an algorithm to recover SiPM signals even when exposed to high light fluxes [16].

\section{Conclusion}

Two SiPM based optical modules have been installed in modified versions of the SSD designed for the Pierre Auger Observatory. It is the first measurement of air showers induced by ultra high energy cosmic ray particles using an SiPM based readout of scintillator detectors. After operation for more than one year in the Argentinean Pampas, they show reliable operation and prove their performance even in such harsh environments. The SiPM gain is stable and single p.e. resolution allows for precise calibration and monitoring of the characteristics of the detector. Coincident measurements with SSDs equipped with conventional PMTs show a good performance of the SiPM based optical modules. Only for very high signals above $\sim 10,000 \mathrm{MIP}$, do they start showing a non- 
linear behavior. The presented optical module proves the performance and maturity of SiPMs also in astroparticle physics applications.

\section{Acknowledgments}

We acknowledge the financial support of the German Federal Ministry of Education and Research. We further thank the Pierre Auger Collaboration and their local staff for their support and assistance during the installation of the SiPM module and for modifying the scintillator detectors to make them usable with the SiPM module.

\section{References}

[1] The Pierre Auger Collaboration, J. Abraham et al., Nucl. Instrum. Meth. A620 (2010) 227-251, [doi:10.1016/j.nima.2010.04.023].

[2] C. Baixeras et al., Nucl. Phys. B (Proc. Suppl.) 114 (2003) 247-252, [doi:10.1016/S0920-5632(02)01910-2].

[3] A. Garcìa et al., PoS (ICRC2019) 267.

[4] The Pierre Auger Collaboration, I. Allekotte et al., Nucl. Instrum. Meth. A586 (2008) 409-420, [doi:10.1016/j.nima.2007.12.016].

[5] HAWC Collaboration, A. U. Abeysekara et al., [arXiv:1310.0074].

[6] IceCube Collaboration, R. Abbasi et al., Nucl. Instrum. Meth. A700 (2013) 188-220, [doi:10.1016/j.nima.2012.10.067].

[7] T. Abu-Zayyad et al., Nucl. Instrum. Meth. A689 (2012) 87-97, [doi:10.1016/j.nima.2012.05.079].

[8] The Pierre Auger Collaboration, J. Schulz, PoS (ICRC2015) 615.

[9] D. Renker and E. Lorenz, JINST 4 (2009) P04004, [doi:10.1088/1748-0221/4/04/p04004].

[10] Hamamatsu Photonics, S13360-6025PE datasheet, June 2019.

[11] T. Bretz et al., JINST 13 (2018) P06001, [doi:10.1088/1748-0221/13/06/p06001].

[12] Hamamatsu Photonics, Technical Information C11204-02, June 2019.

[13] The Pierre Auger Collaboration, A. Aab et al., NIM A798 (2015) 172, [doi:10.1016/j.nima.2015.06.058].

[14] The Pierre Auger Collaboration, R. Smida, PoS (ICRC2017) 390.

[15] The Pierre Auger Collaboration, A. Aab et al., [arXiv:1604.03637].

[16] T. Bretz et al., PoS (ICRC2017) 472. 